\title{
Un hiver pour les livres. Dix lettres de Jean Potocki à Ernst Theodor Langer, bibliothécaire de Wolfenbüttel
}

Lorenz Frischknecht

\section{CpenEdition}

Journals

Édition électronique

URL : http://journals.openedition.org/edl/469

DOI : $10.4000 /$ edl. 469

ISSN : 2296-5084

Éditeur

Université de Lausanne

Édition imprimée

Date de publication : 15 décembre 2012

Pagination : 195-222

ISBN : 978-2-940331-29-1

ISSN : 0014-2026

Référence électronique

Lorenz Frischknecht, « Un hiver pour les livres. Dix lettres de Jean Potocki à Ernst Theodor Langer, bibliothécaire de Wolfenbüttel », Études de lettres [En ligne], 4 | 2012, mis en ligne le 15 décembre 2015, consulté le 21 décembre 2020. URL : http://journals.openedition.org/edl/469; DOI : https://doi.org/ $10.4000 /$ edl. 469 


\section{UN HIVER POUR LES LIVRES. DIX LETTRES DE JEAN POTOCKI À ERNST THEODOR LANGER, BIBLIOTHÉCAIRE DE WOLFENBÜTTEL}

La redécouverte de lettres autographes de Jean Potocki à Ernst Theodor Langer (1743-1820), bibliothécaire de Wolfenbüttel, permet d'accompagner l'érudit pendant ses travaux historiques et géographiques durant l'hiver 1795-1796. Logé à Brunswick, Potocki commande ou renvoie auprès de la bibliothèque une bonne soixantaine d'ouvrages d'auteurs anciens et plus récents. La correspondance permet ainsi d'établir une bibliographie de ses recherches qui aboutissent tout d'abord aux Fragments historiques et géographiques sur la Scythie, la Sarmatie et les Slaves et au Mémoire sur un nouveau péryple du Pont Euxin. En même temps, les lettres nous apportent quelques nouveaux éléments relatifs à la biographie de l'auteur.

Dans son Mémoire sur un nouveau péryple du Pont Euxin, publié en 1796, Jean Potocki insère le fragment d'une carte marine de la Méditerranée et de la Mer Noire qu'il avait découverte dans la bibliothèque de Wolfenbüttel. Il s'explique ainsi concernant son choix:

Plusieurs raisons m'ont portées [sic] a la faire graver de preference aux autres, et j'y ai sur tout été determiné, p[o]ur le desir de temoigner ma reconnoissance à la bibliotheque de Wolfenbutel où mes recherches ont été accueuillies $[s i c]$ avec une prevenance et une hospitalité sans bornes ${ }^{1}$.

I. J. Potocki, Mémoire sur un nouveau péryple du Pont Euxin, ainsi que sur la plus ancienne histoire des peuples du Taurus, du Caucase, et de la Scythie, p. 4. Il s'agit du «Fragment de la carte marine de Fréduce d'Ancona tirée de la bibliothèque de Wolfenbutel ». Cette carte se trouve aussi in fine du tome IV des Fragments historiques 
Une dizaine de lettres de Potocki conservées dans les archives de la Herzog August Bibliothek (HAB) permet de comprendre plus précisément la "prevenance" mentionnée dans ce témoignage. Ce sont des lettres autographes que Potocki avait écrites à Ernst Theodor Langer (1743-1820), bibliothécaire de Wolfenbüttel et successeur de Lessing dans cette fonction après le décès de celui-ci en 1781 .

Une édition antérieure de ces lettres dans une publication germanopolonaise de $1994^{2}$ était passée inaperçue aux yeux de la critique. Il convient, dès lors, de les remettre au jour en profitant de corriger certaines (rares) lacunes dans la lecture du texte, de lire aussi cette correspondance à la lumière des connaissances biographiques acquises depuis et surtout de préciser les nombreuses références bibliographiques auxquelles Potocki ne fait souvent qu'une vague allusion et que l'édition de 1994 omet de développer. Il s'agit d'ouvrages - pour la plupart des livres, mais aussi quelques cartes - que l'érudit, alors en pleines recherches pour le Mémoire sur un nouveau péryple du Pont Euxin et les Fragments historiques et géographiques sur la Scythie, la Sarmatie et les Slaves, commande auprès du bibliothécaire, ou qu'il lui renvoie.

Pour retracer les relations de Potocki avec Wolfenbüttel, on peut d'abord recourir à une autre source, à savoir le registre de prêts. Il permet de conclure que Potocki travaille sur des livres de la bibliothèque au début de l'automne 1795. Selon ce registre, Potocki emprunte deux ouvrages le 15 septembre et deux autres le 19 du même mois ${ }^{3}$. On ignore si le répertoire contient la totalité des prêts pour cette première période, ou si Potocki n'a pas plutôt travaillé, sur place ou à distance, sur un nombre de livres plus grand, peut-être sans les emprunter ou bien sans

et géographiques sur la Scythie, la Sarmatie et les Slaves, publiés la même année. De fait, ce quatrième volume est une réédition des Chroniques, mémoires et recherches pour servir à l'histoire de tous les peuples slaves, imprimées en 1793, ne possédant pas encore cette carte.

2. K. Bednarska-Ruszajowa, "Graf Jan Potocki in der Herzoglichen Bibliothek in Wolfenbüttel».

3. Voir l'analyse de ces livres de prêts dans M. Raabe, Leser und Lektüre im 18. Jahrhundert, p. 339. On trouvera dans les notes de bas de page, au fil de la correspondance, les références exactes de tous les emprunts enregistrés, à deux ouvrages près, car ils ne figurent pas dans les lettres: le 15 septembre, Potocki emprunte: Dionysii Halicarnassei Scripta omnia quae extant, historica et rhetorica, éd. par Friedrich Sylburg, Francfort, 1586; le 19 septembre, il emprunte: Hartmann Schedel, Das buch Der Croniken, Nuremberg, 1493. Les dates de restitution des ouvrages ne sont pas connues. 
que le bibliothécaire les ait enregistrés ${ }^{4}$. Toujours est-il qu'il y aura bientôt une deuxième période de prêts, au début de laquelle Potocki est déjà en mesure de s'orienter très aisément dans les étages de la bibliothèque, puisque dans la première des dix lettres conservées, écrite de Brunswick le 5 décembre 1795, il passe une commande auprès du bibliothécaire Langer sans indication bibliographique, mais en décrivant l'endroit exact où l'ouvrage est placé dans la bibliothèque:

J'aurai besoin de la carte maritime pendue a une fenetre en bas 5 .

Si l'on ne sait pas encore où Potocki a passé les semaines comprises entre septembre et fin novembre ${ }^{6}$, on apprend dans cette lettre qu'il s'était fixé un but précis:

[je su]is venu passer l'hyver a Brunswick, ou je compte [beau]coup sur Wolfenbutel pour ma nourituelle $[s i c]$ spirituelle.

La métaphore consacrée se poursuit lorsque Potocki décrit les circonstances qui le poussent à passer commande à distance:

Mais comme il fait trop froid pour y aller souvent je vous prie de m'envoyer ma provision tout a la fois C'est a dire de prendre tous ces livres chés vous ou j'irai les chercher. moi meme.

On peut lire dans ces phrases une certaine hésitation quant aux modalités. En effet, la livraison des livres se déroulera différemment: Langer propose d'envoyer ces "provisions" directement à Potocki, toujours logé à Brunswick.

Cette lettre du 5 décembre 1795 est le point de départ d'un échange à haute fréquence entre Potocki et Langer durant l'hiver ${ }^{7}$. Les lettres

4. Il convient de lire le registre de la bibliothèque avec certaines réserves. Le répertoire ne mentionne que cinq autres emprunts de Potocki en décembre et en janvier, tandis que les lettres écrites à Langer prouvent qu'il s'agit d'un nombre d'ouvrages plus élevé. En outre, K. Bednarska-Ruszajowa souligne que les emprunts ne sont pas classés selon la date de l'emprunt, mais selon les retours, ce qui laisserait planer un doute sur la crédibilité de cette source concernant le séjour de Potocki ( Graf Jan Potocki in der Herzoglichen Bibliothek in Wolfenbüttel», p. 33).

5. Voir Annexe I, Lettre I.

6. Il est à Brunswick le $1^{\text {er }}$ décembre, puisqu'il y écrit à Stanislas Félix Potocki une lettre datée de ce jour (J. Potocki, Euvres, V, p. 44-46).

7. On ignore si les relations avec Langer dépassaient le cadre des emprunts. Il faut rappeler que Langer avait demandé dans son testament "d'anéantir et de brûler» la 
suivantes (II-IX) datent du 8 décembre et du 14 décembre, du 6 janvier, du 27 janvier, du 12 février, du 20 février ainsi que du $1^{\text {er }}$ mai (toutes écrites de Brunswick) et du 14 juin (écrite de Berlin). Cette concentration temporelle de la correspondance laisse supposer que la "prevenance» soulignée par Potocki est liée aussi à la grande disponibilité du bibliothécaire. Une seule lettre de Potocki ne porte ni date ni indication de lieu (X). Bien qu'elle soit conservée dans les archives au milieu des autres lettres, elle ne s'insère pas dans cet échange de livres; il s'agit d'une lettre de recommandation. Il faut ajouter en outre la lettre du 6 janvier 1797, déjà éditée ${ }^{8}$, dont le destinataire a été identifié comme étant l'imprimeur Friedrich Vieweg (1761-1835). Elle est conservée sous une cote différente (V 1221).

Les lettres de décembre 1795 contiennent des listes d'œuvres que Potocki veut emprunter. A partir de janvier, l'auteur énumère également celles qu'il renvoie à la bibliothèque. La chronologie des prêts montre que Potocki garde ces livres pendant peu de semaines, ce qui témoigne d'un rythme de travail très intense. La correspondance de cet hiver 1795-1796 permet donc d'établir une bibliographie des ouvrages consultés - non exhaustive - pour le Mémoire sur un nouveau péryple du Pont Euxin où Potocki donne lui-même des références au fonds de Wolfenbüttel, et surtout pour les Fragments historiques et géographiques, qui consistent en une compilation de nombreuses sources. Il s'agit de 57 ouvrages au total : 28 ouvrages pour lesquels l'emprunt, la restitution ou la simple consultation sont attestés, et 29 ouvrages pour lesquels seule la commande est prouvée. On trouvera cette «bibliographie» dans l'annexe de cette communication. Elle consiste d'abord en un grand nombre d'auteurs latins et grecs, en grande partie des historiens et des géographes de l'Antiquité tardive. Ensuite, ce sont des érudits plus récents de ces mêmes disciplines, ainsi que des philosophes, des linguistes ou des voyageurs. Nombreux sont les auteurs dont Potocki emprunte ou commande les ouvrages auprès de Wolfenbüttel, et qu'il citera effectivement dans ses Fragments historiques et géographiques. Dans la «Table des auteurs

totalité de ses lettres et écrits. Selon toute vraisemblance, son vœu a été exaucé. Seule la correspondance officielle a été sauvée, comme l'atteste son biographe (P. Zimmermann, Ernst Theodor Langer, Bibliothekar in Wolfenbüttel, ein Freund Goethes und Lessings, p. 8).

8. J. Potocki, Euvres, V, p. 51. 
extraits" qui conclut l'introduction de cette œuvre, on retrouve un certain nombre des emprunts faits au fonds de Wolfenbüttel, mais pas tous. La bibliothèque de Wolfenbüttel n'a pu fournir qu'une partie des sources nécessaires; les autres livres auront sans doute été trouvés dans plusieurs bibliothèques (dont des bibliothèques privées) dans lesquelles il travaillait au cours de son séjour allemand.

Parfois, Potocki semble bien savoir ce qu'il cherche, car il indique pour certaines ouvres quelle édition et dans quel format ou bien quelle traduction il souhaite lire. Or pour la plupart des commandes, ces références sont très rudimentaires; Potocki nomme tantôt l'auteur, tantôt le titre, et ceci presque toujours en abrégé. C'est pourquoi il n'est pas possible d'identifier les éditions pour la totalité des références. Il faut dire aussi que pour une grande partie de ces œuvres, on n'a pas de trace qui permettrait de savoir que la commande a été réalisée par le bibliothécaire. Car vers la fin de son séjour à Brunswick, Potocki n'indique plus en détail quels livres il renvoie. Ainsi, dans la lettre du $1^{\text {er }}$ mai 1796 , il ne mentionne que globalement les retours:

J'ai l'honneur de vous envoyer ci joints tous les livres que j'ai eu de la bibliotheque aussi bien que les cartes a l'exception de $[\ldots]^{9}$.

En effet, le registre de prêts contient, pour la date du 11 mai, quatre œuvres rendues ${ }^{10}$; évidemment, il ne peut pas s'agir de la totalité. Le dernier retour est signalé le 28 juillet de la même année.

Outre ces indications concernant les prêts, les lettres à Langer permettent d'ajouter certains aspects à la biographie de Potocki et de remplir des lacunes dans la chronologie de ces quelques mois. On dispose ainsi d'une source supplémentaire qui vient compléter celles déjà connues, qui sont le registre de prêts, une brève mention par le marquis de La Maisonfort (1763-1827) ${ }^{11}$ et d'autres correspondances. On

9. Annexe I, Lettre VIII.

Io. Concernant ces dates, nous suivons la lecture de K. Bednarska-Ruszajowa ("Graf Jan Potocki in der Herzoglichen Bibliothek in Wolfenbüttel», p. 30-32) puisque ses indications se confirment dans les lettres à Langer. Cette lecture s'oppose à celle de M. Raabe qui indique le 2 mai pour la date de l'emprunt de ces ouvrages et ne mentionne pas celle du retour (Leser und Lektüre im 18. Jahrhundert, p. 339).

II. "J'avais retrouvé à Florence [en 1803] un comte Jean Potocki que j'avais beaucoup vu à Brunswick [en 1795-1796]" (La Maisonfort, Mémoires d’un agent royaliste sous la Révolution, l'Empire et la Restauration 1763-1827, p. 302). 
apprend ici que Potocki fréquentait les milieux intellectuels de la ville de Brunswick: non seulement il était en contact avec le Duc Karl Wilhelm Ferdinand von Braunschweig-Wolfenbüttel (1735-1806), à qui il a prêté lui-même au moins un des livres empruntés (I et IX), mais il logeait aussi à l'Hôtel d'Angleterre, l'hôtel le plus distingué de Brunswick, où était domicilié le "Grosser Club", une société réunissant l'élite intellectuelle de la ville et offrant à ses membres un salon de lecture doté de journaux historiques et politiques (II). Il est possible que Jean Potocki y ait rencontré aussi un certain Monsieur Dolomieu, émigré français ou flamand, de la part de qui il rend trois livres à la bibliothèque (IV). Les lettres permettent également de dater plus précisément le départ de Potocki. Le $1^{\mathrm{er}}$ mai, il annonce qu'il va venir prendre congé chez Langer (VIII). Le 15 mai, il écrit encore une lettre de Brunswick ${ }^{12}$. Le 14 juin, il se trouve déjà à Berlin (IX); jusqu'ici, le jour le plus tardif envisagé pour son arrivée à Berlin était le 10 juillet, date de la lettre à Platon Alex. Zoubov (1767-1822) ${ }^{13}$.

Enfin, les lettres confirment que Potocki aimait échanger les informations et les savoirs autant que possible, et remonter directement à tout ouvrage propre à lui fournir de nouvelles connaissances. Ainsi, il remercie Langer d'une indication concernant des dissertations de l'historien allemand Johann Christoph Gatterer (1727-1799) et plus précisément son "opinion [...] au sujet des Sarmates" (V). En effet, Potocki mentionnera Gatterer dans le premier tome des Fragments historiques et géographiques en laissant entrevoir un certain espoir de voir ses propres travaux agréés par l'érudit allemand:

[...] sans doute l'on doit tout attendre d'un savant tel que [Gatterer], surtout l'on à $[s i c]$ droit d'esperer, qu'il voudra bien justifier pleinement les nouvelles leçons qu'il dicte, dans la lecture de plusieurs anciens, et qu'il auroit sans doute développé davantage, s'il ne s'étoit trouvé restreint dans les bornes d'un memoire accademique ${ }^{14}$.

Potocki citera plusieurs fois Gatterer au cours de ses propres recherches historiques, surtout quand il s'agit de la question de savoir si les Sarmates

I2. Lettre à Jacques de la Gardie (in J. Potocki, Euvres, V, p. 46 sq.).

I3. J. Potocki, Euvres, V, p. 47 sq.

I4. J. Potocki, Fragments historiques et géographiques sur la Scythie, la Sarmatie et les Slaves, p. 6. 
sont des Slaves ou non ${ }^{15}$. Il semble donc que ces lettres écrites au bibliothécaire de Wolfenbüttel témoignent aussi des débuts de son intérêt pour les écrits de cet érudit.

Les dix lettres autographes de Jean Potocki à Ernst Theodor Langer sont conservées par la Herzog August Bibliothek à Wolfenbüttel sous la cote BA II 110, fol. 179-198 (à l'exception du fol. 182 qui contient une liste d'œuvres géographiques en Kurrentschrift allemande qui n'est pas de la main de Potocki; il s'agit sans doute de notes de Langer). Potocki attribue à Langer les titres de "conseiller privé", "garde de bibliothèque", "bibliothécaire" dans les adresses. Si un ouvrage, un auteur ou une édition ne sont pas identifiés, cela est indiqué dans les références bibliographiques. Pour les indications tirées des registres des prêts, je renvoie ici à K. Bednarska-Ruszajowa, "Graf Jan Potocki in der Herzoglichen Bibliothek in Wolfenbüttel», p. 30-32, sans l'indiquer à chaque occurrence.

Je tiens à remercier les conservateurs de la Herzog August Bibliothek pour leur aide. J'exprime aussi toute ma gratitude à Raoul Richner, collaborateur scientifique à l'édition de la collection Zurlauben (Aarau), pour son aide précieuse concernant les références bibliographiques.

\section{Lorenz FRISCHKNECHT}

Zurich/Montpellier, IRCL (Institut de Recherche sur la Renaissance, l'âge Classique et les Lumières)

15. Potocki rend d'abord hommage à Gatterer dans la lettre du 10 juillet 1796 à Platon Alex. Zoubov (Euvres, V, p. 48); ensuite, il y a un renvoi dans le Voyage du comte Jean Potocki à Astrakhan et dans les cantons voisins, en 1797 (Euvres, II, p. 185); on en trouve enfin plusieurs citations dans l'Histoire primitive des peuples de la Russie avec une exposition complète de toutes les notions, locales, nationales et traditionnelles, nécessaires à l'intelligence du Quatrième Livre d'Hérodote. 


\section{ANNEXE I}

L'édition se conforme aux principes des Euvres éditées par François Rosset et Dominique Triare, avec respect rigoureux des graphies et de la ponctuation.

\section{Lettre I}

[181r]

Brunswick. le. Samedi 5. decembre 1795.

Monsieur.

La lettre que j'ai l'honneur de vous ecrire est [d'a]bord pour vous faire mon compliment ayant apris [que] votre santé étoit bonne. et vous anocer que [je su] is venu passer l'hyver a Brunswick, ou je compte [beau-] coup sur Wolfenbutel pour ma nourituelle [sic] spirituelle

Mais comme il fait trop froid pour y aller souvent je vous prie de m'envoyer ma provision tout a la fois C'est a dire de prendre tous ces livres chés vous ou j'irai les chercher. moi meme.

Outre les livres, j'aurai besoin de la carte ge ${ }^{16}$ maritime pendue a une fenetre en bas ${ }^{17}$, et aussi du petit atlas, sur velin ${ }^{18}$. pour lesquels je vous

16. Biffé.

17. Selon le registre de prêts, Potocki emprunte, du 10 décembre 1795 au 11 mai 1796, Eine gezeichnete Karte Europae et Asiae - conf. eine grosse Pergamenthaut, s. 1., s. d. (Lettre VIII). Il s'agit sans doute de: Ottomanno Freducci, Portulankarte des MittelMeeres mit ganz Europa und Africa/Nord, s. 1., 1497 (voir K. Bednarska-Ruszajowa, "Graf Jan Potocki in der Herzoglichen Bibliothek in Wolfenbüttel», p. 32), c'est-à-dire de la carte mentionnée dans le Mémoire sur un nouveau péryple du Pont Euxin, p. 4, et dont un fragment est inséré à la fois dans cet ouvrage et dans les Fragments historiques et géographiques sur la Scythie, la Sarmatie et les Slaves.

I8. Selon le registre de prêts, Potocki emprunte, du 10 décembre 1795 au 11 mai 1796, Ein Atlas von 15 Pergamentblättern, s. l., s. d. (Lettre VIII). Il s'agit sans doute du Seeatlas. Ein Atlas von 14 Doppelblättern plus letztes Blatt aus Pergament, Venise, 1554 (voir K. Bednarska-Ruszajowa, "Graf Jan Potocki in der Herzoglichen Bibliothek in 
porterai une permission du Duc ${ }^{19}$, et s'il y a d'autres cartes manuscriptes ou bien tres anciennes je vous prie de me les donner egalement. Je suis avec une considération parfaite Monsieur

Votre tres humble Serviteur le Comte J. Potocki

Vous trouverés sur l'autre feuille les livres dont j'ai besoin et que je viendrai chercher chés vous.

[181v: blanc]

[183r]

De la bibliotheque Byzantine. Theophane, Anastase et Nicephore ${ }^{20}$.

Carpzow neuerofneter Ehrentempel merkwurdiger antiquitaten des Marckgraftums oberlausitz. Von Joh. Benedict Carpzow. Leipzig und Budissin 1719. fol ${ }^{21}$.

Walwassor, descript. Carniae, 4 vol fol ${ }^{22}$

Duysbourg apud Hartcknochium. 1 vol. $4^{\circ} 23$

Scriptores rerum Brunswiciens. Leibnitzii 3 vol. fol ${ }^{24}$.

Deguignes. Hist. des huns ${ }^{25}$.

Wolfenbüttel ", p. 31), c'est-à-dire de la carte mentionnée comme une des sources dans le Mémoire sur un noveau péryple du Pont Euxin, p. 5. On y trouve aussi (p. 4 sq.) la référence à une troisième carte de Wolfenbüttel, préparée par Battista Agnese à Venise en 1514. Ces trois cartes sont probablement celles que Jean Potocki évoque dans sa lettre à Stanislas Félix Potocki le $1^{\text {er }}$ décembre, donc peu de jours avant cette commande: "j'ai encore fait une découverte important[e] dans la bibliotheque de Wolfenbuttel. Ce sont des cartes tres anciennes sur velin, ou il y a bien des choses sur la mer noire" (Euvres, V, p. 45).

19. Karl Wilhelm Ferdinand von Braunschweig-Wolfenbüttel (1735-1806).

20. Théophane le Confesseur, Chronographia, éd. non identifiée; Anastase le Bibliothécaire, Chronographia tripartita, éd. non identifiée; Nicéphore de Constantinople, Chronographia, éd. non identifiée.

2I. Johann Benedict Carpzov, Neueröffneter Ehren-Tempel Merckwürdiger Antiquitäten des Marggraffthums Ober-Lausitz, Leipzig/Budissin, 1719 (Lettre V).

22. Johann Weikhard von Valvasor, Die Ehre deß Hertzogthums Crain [...], Laybach, 1689 (Lettres III et V).

23. Petrus de Dusburg, Chronicon Prussiae, éd. par Christoph Hartknoch, Francfort/ Leipzig/Jena, 1679 (Lettres III et IV).

24. Gottfried Wilhelm Leibniz, Scriptores rerum Brunsvicensium, Hannovre, 17071711 (Lettre IV).

25. Joseph de Guignes, Histoire générale des Huns, des Turcs, des Mogols et des autres Tartares occidentaux, Paris, 1756-1758. 
Luc. Apuleius _ opera omnia qui ${ }^{26}[$ sic $]$ extant $^{27}$

Diogene Laerce et une traduction françoise s'il y en a une ${ }^{28}$

Paul Warnefried. historia longobardorum ${ }^{29}$

Abulpharage texte syriaque avec la version latine ${ }^{30}$

Ramusio viaggi. en italien 3 vol. fol ${ }^{31}$

Bergeron recueuil [sic] de voyages 2 vol. in $4^{\circ} 32$

Assemani, calendaria eclesiae slavorum 4 vol. in $4^{\circ 33}$

Cassidorus epistolae ${ }^{34}$

L'art de verifier les dates ${ }^{35}$

Tablettes chronologiques de Langlet du Frenoy ${ }^{36}$ Alcaeus. apud Fulvium Ursinum ou ailleurs ${ }^{37}$.

[183v: Adresse et sceau]

26. Biffé: que [sic].

27. Apulée, Apuleii Madaurensis Platonici Opera Omnia quae exstant, éd. non identifiée (Lettre IV).

28. Diogène Laërce, De vitis, dogmatibus et apophtegmatibus clarorum philosophorum libri X, éd. non identifiée; trad. anonyme, Les vies des plus illustres philosophes de l'antiquité [...], Amsterdam, 1758.

29. Paul le Diacre, De gestis Langobardorum libri VI, éd. non identifiée.

30. Probablement Barhebraeus, Chronicon Syriacum, éd. par Georg Wilhelm Kirsch, Leipzig, 1789. Barhebraeus est une forme latinisée du nom d'Aboul Faradj. Le 6 janvier, Potocki renvoie un autre ouvrage du même auteur: Specimen Historiae Arabum, Oxford, 1650 (Lettre IV).

3I. Giovanni Battista Ramusio, Navigationi e viaggi [...], éd. non identifiée

32. Pierre de Bergeron (éd.), Voyages faits principalement en Asie dans les XII, XIII, $X I V$, et XV siecles, La Haye, 1735. Le 6 janvier, Potocki renvoie Pierre de Bergeron (éd.), Les Voyages Fameux du Sieur Vincent Le Blanc Marceillois [...], Paris, 1649 (Lettre IV).

33. Giuseppe Simone Assemani, Kalendaria ecclesiae universae [...], Kalendaria ecclesiae Slavicae sive Graeco-Moscha, Rome, 1755 (Lettre IV).

34. Cassiodore, Variae epistolae, éd. non identifiée (Lettre V).

35. Maur François Dantine, Ursin Durand, Charles Clémencet, L’Art de vérifier les dates des faits historiques, des chartes, des chroniques, et autres anciens monumens [...], Paris, 1750 ; nv. éd. revue et augmentée, Paris, 1770. Selon le registre de prêts, Potocki emprunte ce livre le 15 septembre 1795; la date du retour n'est pas indiquée.

36. Nicolas Lenglet-Dufresnoy, Tablettes chronologiques de l'histoire universelle sacrée et profane, ecclésiastique et civile [...], éd. non identifiée.

37. [Alcaeus et al.], Carmina novem illustrium feminarum [...], éd. par Fulvio Orsini, Anvers, 1568 (Lettre VIII). Selon le registre de prêts, Jean Potocki emprunte ce livre du 10 décembre 1795 au 11 mai 1796. 


\section{Lettre II}

[186r]

\section{Monsieur}

Mon intention étoit d'aller moi meme a Wolfenbutel chercher les livres que vous me prometés et qui s'y trouven $[s i c]$ mais puisque vous avés la complaisance de vouloir bien me les envoyer a mon Hotel d'Angleterre chés Renckendorf ${ }^{38}$, je vous en suis obligé et les atendrai de pied ferme. mille graces pour votre obligeance

38. Rüttger Heinrich Rönkendorf était le patron et propriétaire de l'Hôtel d'Angleterre, l'hôtel le plus distingué de Brunswick, ouvert en 1778 et situé à la Breite Strasse 18. Autour de 1780, Rönkendorf y fonda le "Grosser Club", réunissant bientôt l'élite intellectuelle de la ville (professeurs du Collegium Carolinum, officiers de la cour, marchands fortunés). Gotthold Ephraim Lessing (1729-1781) aurait participé à la fondation, et l'écrivain Johann Anton Leisewitz (1752-1806) aurait également compté parmi les membres. Selon les statuts de 1813, le but du club consistait en «la distraction agréable et utile, l'instruction scientifique et le divertissement en société»; on peut supposer que c'était le cas déjà lors de la visite de Potocki ("nützliche und angenehme Unterhaltung, wissenschaftliche Belehrung und geselliges Vergnügen», je traduis des Statuten des grossen Clubbs zu Braunschweig, p. 3). Le "Grosser Club» hébergeait un salon de lecture doté tout d'abord de journaux historiques et politiques et, à partir de la deuxième moitié du XIX ${ }^{\mathrm{e}}$ siècle, d'œuvres érudites et littéraires, de revues et de cartes. En 1921, un fonds comportant 1500 ouvrages fut intégré dans la bibliothèque de la ville de Brunswick. L'Hôtel d'Angleterre accueillit d'autres voyageurs, parmi eux Karl Gottlob Küttner (1755-1805) qui dans ses rapports de voyages le qualifie de «sehr gut» (Reise durch Deutschland, Dänemark, Schweden, Norwegen und einen Theil von Italien in den Jahren 1797, 1798, 1799, t. I, p. 464). On peut mentionner aussi Jean-Pierre ou Nicolas-François Blanchard (1753-1809). Il logeait à l'hôtel d'Angleterre en 1788 pour se préparer à son $32^{\mathrm{e}}$ voyage en ballon, qui eut lieu à Brunswick le 30 août de la même année. C'est-à-dire que Blanchard avait séjourné à Brunswick peu avant son arrivée à Varsovie où il effectua son $36^{\mathrm{e}}$ vol le 14 ou le 16 mai 1790 , avec la participation de Jean Potocki qui s'embarqua lui-même avec Blanchard dans la machine aérostatique. Blanchard a-t-il recommandé à Potocki l'Hôtel d'Angleterre? Ces indications sont tirées des sources suivantes: Braunschweiger Tageblatt, 454 (27 septembre 1890); avis lors du centenaire de l'hôtel dans Braunschweigische Anzeigen, 27 juillet 1878; E. Schulz, So sahen wir Braunschweig, p. 131-133; L. Camerer et al., Braunschweiger Stadtlexikon, p. 92, cité sur le site internet de la Bibliothèque municipale de Brunswick, www. braunschweig.de/kultur_tourismus/bibliotheken_archive/stadtbibliothek/sammlungen.html [30 avril 2012] ; P. Raabe (Hrsg.), Handbuch der historischen Buchbestände in Deutschland, p. 55; D. Diestelmann, Kleine Braunschweiger Stadtgeschichte, p. 9. Je remercie le Staatsarchiv Wolfenbüttel et le Staatsarchiv Braunschweig de m’avoir fourni quelques-unes de ces sources. 
Le Comte Jean Potocki

Brunswick. le mardi 8 [décembre 1795].

[186v: Adresse et sceau]

\section{Lettre III}

[184r]

Brunswic le lundi. 14. Decembre. 1795.

\section{Monsieur}

J'ai bien des graces a vous rendre de votre exactitude et la richesse de la bibliotheque m'inspire une [v]éritable admiration sur tout a l'égard des [car]tes sur velin dont j'espere tirer le plus [grand] parti. Apresent voici encore les choses que [je veux] vous demander.

1. Les trois volumes restants de Walwassor [dont] vous ne m'avés envoyé qu'un ${ }^{39}$.

2. Je voudrais bien avoir une certaine edition de Duysbourg qui est accompagnée d'un grand nombre de dissertations. et entre autres une sur la langue, ou se trouve un pater noster prussien ${ }^{40}$. Si je pouvais avoir quelque collection de pater noster [c]ela me feroit beaucoup de plaisir ${ }^{41}$.

3. Herbelot, bibliotheque orientale ${ }^{42}$

Voila tout pour le moment. Je suis avec [u]ne considération parfaite. Monsieur

39. Johann Weikhard von Valvasor, Die Ehre deß Hertzogthums Crain [...], Laybach, 1689 (Lettres I et V).

40. Petrus de Dusburg, Chronicon Prussiae, éd. par Christoph Hartknoch, Francfort/ Leipzig/Jena, 1679 (Lettres I et III).

4I. Gustav von Bergmann (Hrsg.), Das Gebeth des Herrn, oder Vaterunsersammlung in 152 Sprachen, Ruien, 1789 (Lettre VIII). Selon le registre de prêts, Potocki emprunte ce livre du 16 décembre 1795 jusqu'au 11 mai 1796. Potocki insère une partie de ces pater noster dans le premier tome des Fragments historiques et géographiques sur la Scythie, la Sarmatie et les Slaves, p. 36-38, sous le titre "Oraisons dominicales dans tous les dialectes Slaves, tirées de la collection de Bergmann, imprimé[e] à Ruien en Livonie en l'année 1789».

42. Barthélemy d'Herbelot de Molainville, Bibliothèque orientale ou Dictionnaire universel contenant generalement tout ce qui regarde la connoissance des peuples de l'Orient [...], Paris, 1697. 
Votre tres humble et Tres obeïssant Serviteur

Le Comte Jean Potocki

[184v: Adresse et sceau]

\section{Lettre IV}

[188r]

Monsieur.

Comme vous m'avés marqué le desir de ravoir aussitot que possible, les Scriptores rerum Brunswiciensium ${ }^{43}$ je m'empresse de vous les renvoyer. et j'y joins

1. Le Specimen historiae Arabum ${ }^{44}$.

2. Bergeron voyages de le Blanc ${ }^{45}$.

3. Apulée ${ }^{46}$.

4. Scriptores diversorum gentium ${ }^{47}$

5. Duysbourg, Chronicon Prussiae ${ }^{48}$.

6. Calendaria eclesiae Slavorum ${ }^{49}$

Et de la part de Monsieur de Dolomieux ${ }^{50}$.

43. Gottfried Wilhelm Leibniz, Scriptores rerum Brunsvicensium, Hannovre, 17071711 (Lettre I).

44. Barhebraeus, Specimen Historiae Arabum, Oxford, 1650. En décembre, Potocki avait commandé un autre ouvrage de l'auteur: Chronicon Syriacum, Leipzig, 1789 (Lettre I).

45. Pierre de Bergeron (éd.), Les Voyages Fameux du Sieur Vincent Le Blanc Marceillois [...], Paris, 1649. Potocki avait commandé en décembre un "recueuil [sic] de voyages» édité par Bergeron (Voyages faits principalement en Asie dans les XII, XIII, XIV, et XV siecles, La Haye, 1735; Lettre I).

46. Apulée, Apuleii Madaurensis Platonici Opera Omnia quae exstant, éd. non identifiée (Lettre I).

47. Ouvr. non identifié et biffé.

48. Petrus de Dusburg, Chronicon Prussiae, éd. par Christoph Hartknoch, Francfort/ Leipzig/Jena, 1679 (Lettres I et III).

49. Giuseppe Simone Assemani, Kalendaria ecclesiae universae [...], Kalendaria ecclesiae Slavicae sive Graeco-Moscha, Rome, 1755 (Lettre 1).

50. Dans son répertoire à partir des registres des prêts, $M$. Raabe mentionne un Monsieur Dolomieu en indiquant qu'il est membre des émigrés français et flamands (Leser und Lektüre im 18. Jahrhundert, p. 87). On apprend également qu'il avait emprunté, le 10 octobre 1795, les trois ouvrages qui suivent dans la lettre de Potocki. 
1. La combe. abrégé Chronologique de l'hist. du N. volume. 1. et $2^{51}$

2. Annales d'Italie tome. $12^{52}$.

3. Hist. literaire de la France. Tome. $7^{53}$.

Je vous avois marqué que je n'aurois plus besoin d'autres livres pour cet hyver. Mais ma Minerve en ordone autrement. Et j'entreprens encore l'impression d'un volume qui completera mon histoire des Slaves depuis l'Expedition de Darius jusques a l'année 900 de J.C.

J'aurai donc besoin de nouveaux volumes, et je vous demande pour le moment

[188v]

1. La table Peutigerienne ${ }^{54}$.

2. Les Geographi minores, 4 vol. in 8. Graeci ${ }^{55}$

3. Paul Orose, et Jule Solin ${ }^{56}$

4. Antoni itinerarium ${ }^{57}$.

5. Scriptores Historiae Augustae Sylburgii ${ }^{58}$

6. Ptolemée ${ }^{59}$.

7. Deux peryples dont l'un doit être de Scylax de Cariandre et l'autre anonyme, edités je crois, par Vossius ${ }^{60}$.

5I. Jacques Lacombe, Abrégé chronologique de l'histoire du Nord, Amsterdam, 1763.

52. Ludovico Antonio Muratori, Annali d'Italia dal principo dell'era volgare sino l'anno 1500, Milan, 1744-1749.

53. Selon le registre de prêts, Potocki avait emprunté lui-même le 19 septembre 1795 les tomes I et VIII de Dom Antoine Rivet de la Grange, Histoire littéraire de la France [...], éd. par la Congrégation de Saint-Maur, Paris, 1733. La date du retour n'est pas indiquée.

54. Ed. non identifiée.

55. John Hudson (ed.), Geographiae veteris Scriptores Graeci Minores, Oxford, 16981717.

56. Paulus Orosius, Adversus paganos Historiarum libri septem, éd. non identifiée; Caius Julius Solinus, Polyhistor, éd. non identifiée (Lettre VI).

57. Itinerarium Antonini Augusti, éd. non identifiée.

58. Friedrich Sylburg (ed.), Historiae Augustae Scriptores Latini Minores [...], Francfort, 1588 (Lettre VI).

59. Claude Ptolémée, Cosmographia, éd. non identifiée; trad. Géographie, éd. non identifiée (Lettre VI).

6o. Isaac Vossius (éd.), Periplus Scylacis Caryandensis [...] ; Accedit Anonymi Periplus [...], Amsterdam, 1639. 
8. Pomponius, Mela. Avienus. et Pline la Geographie seulement ${ }^{61}$ Item.

Hermite le Soulier, extrait nobiliaire de la touraine, Bery, Anjou $\&^{62}$ Mariano, histoire d'Espagne traduite en francois ${ }^{63}$

Mallet, histoire de Dannemarc ${ }^{64}$

Je me recomande a votre patience, et suis avec une considération parfaite Votre tres humble et tres obeissant Serviteur.

Le Comte Jean Potocki

Brunswick le. 6 janvier. 1796.

P.S. bien des compliments pour la nouvelle année.

[189r: blanc]

[189v: Adresse et sceau; note postale écrite d'une autre main $\left.{ }^{65}\right]$

\section{Lettre V}

[190r]

Brunswick. le 27. janvier 1796.

Je vous rends mille graces tant pour le dernier envoy que pour l'avis au sujet des dissertations de Gatterer ${ }^{66}$. que j'ai aussitot demander [sic] a Gotingue. Je connoissois l'opinion de Gatterer au sujet des Sarmates, et

6I. Pomponius Mela, De situ orbis libri tres, éd. non identifiée; Rufus Festus Avienus, Descriptio orbis terrae, éd. non identifiée; Pline l'Ancien, Naturalis historia, éd. non identifiée; trad. Histoire naturelle, éd. non identifiée (Lettre VI).

62. Jean-Baptiste L'Hermite-Souliers, Histoire généalogique de la noblesse de Tourraine [...], Paris, 1665 .

63. Juan de Mariana, Histoire générale d'Espagne [...], trad. par Jean-Baptiste Morvan de Bellegarde, Paris, 1723.

64. Paul Henri Mallet, Histoire de Dannemarc, Copenhague, 1755-1765 (Lettre VI).

65. Note en Kurrentschrift allemande: "ein pak mit bücher gezeichnet» ["accompagnant un paquet de livres», je traduis].

66. Johann Christoph Gatterer (1727-1799). Dans Einleitung in die synchronistische Universalhistorie, t. 2, p. 825-831, l'historien allemand consacre tout un chapitre aux Sarmates. Partant d'une citation de Tacite, Gatterer réfléchit sur la question de savoir s'il faut compter les Peuciens, les Vénèdes et les Finnois parmi les Germains ou parmi les Sarmates. 
c'étoit assés la mienne. mais je n’avois jamais été content de mes preuves, et je suis curieux de voir les Siennes. Je vous renvoye ci joint

Carpzow 1 vol. in fol ${ }^{67}$.

Walwassor 5 vol. in fol ${ }^{68}$.

Cassiodore 1 vol. in $12^{69}$.

Symmache 1 vol. in $12^{70}$.

Theoremata 1 vol. in $4^{\circ} 71$.

Ayés la bonté de m'envoyer Etienne de Bysance ${ }^{72}$ Beauplan description d'Ukranie ${ }^{73}$ (s'il se trouve) et les revolutions d'Italie de Denina traduction de Jardin ${ }^{74}$.

Je suis avec une estime parfaite. Monsieur

V. t. H. et t. ob Servit

Le Comte Jean Potocki

[190v et 191r: blancs]

[191v: Adresse et sceau; note postale écrite d'une autre main ${ }^{75}$ ]

\section{Lettre VI}

$[179 \mathrm{r}]$

Brunswick. le 12. Fevrier. 1796.

67. Johann Benedict Carpzov, Neueröffneter Ehren-Tempel Merckwürdiger Antiquitäten des Marggraffthums Ober-Lausitz, Leipzig/Budissin, 1719 (Lettre I).

68. Johann Weikhard von Valvasor, Die Ehre deß Hertzogthums Crain [...], Laybach, 1689 (Lettres I et III).

69. Cassiodore, Variae epistolae, éd. non identifiée (Lettre I).

70. Probablement Quintus Aurelius Symmaque, Epistolarum libri decem, Leyde, 1653. Parmi les nombreuses éditions conservées à Wolfenbüttel, c'est la seule qui corresponde au format indiqué par Potocki.

7I. Ouvr. non identifié.

72. Stephanus Byzantinus, Ethnika kat' epitomen, éd. non identifiée.

73. Guillaume le Vasseur de Beauplan, Description d'Ukranie qui sont plusieurs Provinces du Royaume de Pologne, Rouen, 1660 (Lettres VIII et IX). Selon le registre de prêts, Potocki emprunte cet ouvrage du 30 janvier au 28 juillet 1796.

74. Carlo Denina, Delle Rivoluzioni d'Italia Libri Ventiquattro, Turin, 1769-1770; trad. par Abbé Jardin, Révolutions d'Italie, Paris, 1771-1775.

75. Note en Kurrentschrift allemande: "ein pak mit bücher gezeichnete» [«accompagnant un paquet de livres», je traduis]. 
Monsieur.

J'ai l'honneur de vous renvoyer ci joint les livres suivants

Zosime. in $8^{76}$.

historia augusta. in $8^{\circ 77}$.

Solinus, polihistor. in $8^{78}$.

Aurelius victor. in $8^{79}$.

Mallet. _- in $4^{\circ} 80$.

Ptolemée. __ in fol ${ }^{81}$.

En revanche je vous prie de m'envoyer.

Strabon ${ }^{82}$.

Herodote ${ }^{83}$.

Diod. Sicul ${ }^{84}$.

Theucidide ${ }^{85}$.

Le reste de Pline ${ }^{86}$.

Ammien Marcellin ${ }^{87}$.

Hypocrate de aere et Humido ${ }^{88}$.

Je suis avec une parfaite consideration. Votre Tres humble serviteur

76. Le catalogue de Wolfenbüttel contient une édition in $12^{\circ}$ : Xiphilin, Zonare, Zosime, Histoire romaine, trad. par Louis Cousin, Paris, 1686.

77. Friedrich Sylburg (ed.), Historiae Augustae Scriptores Latini Minores [...], Francfort, 1588 (Lettre IV).

78. Caius Julius Solinus, Polyhistor, éd. non identifiée (Lettre IV).

79. Aurelius Victor, ouvr. non identifié.

8o. Paul-Henri Mallet, Histoire de Dannemarc, Copenhague, 1755-1765 (Lettre IV).

8I. Claude Ptolémée, Cosmographia, éd. non identifiée; trad. Géographie, éd. non identifiée (Lettre IV).

82. Strabon, Geôgraphiká, éd. non identifiée; trad. La Géographie, éd. non identifiée.

83. Hérodote, Historíai, éd. non identifiée; trad. Les histoires, éd. non identifiée (Lettre VII).

84. Diodore de Sicile, Bibliothéke historiké, éd. non identifiée; trad. La bibliothèque des histoires, éd. non identifiée (Lettre VII).

85. Thucydide, Historía tou Peloponnesiakoú polémou, éd. non identifiée; trad. Histoire de la guerre du Péloponnèse, éd. non identifiée.

86. Pline l'Ancien, Naturalis historia, éd. non identifiée; trad. Histoire naturelle, éd. non identifiée (Lettre IV).

87. Ammianus Marcellinus, Res gestae ou les dixhuit livres de son histoire qui nous sont restés, trad. Guillaume de Moulines, Berlin, 1775. Potocki mentionne cette traduction dans les Fragments historiques et géographiques sur la Scythie, la Sarmatie et les Slaves, t. I, p. 35.

88. Hippocrate de Cos, De aere, aquis et locis opus, éd. non identifiée. 
Le Comte Jean Potocki

$[179 \mathrm{v}$ et $180 \mathrm{r}$ : blancs]

[180v: Adresse et sceau; note postale écrite d'une autre main $\left.{ }^{89}\right]$

\section{Lettre VII}

[194r]

\section{Monsieur}

J'ai l'honneur de vous remercier de l'envoy que vous m'avés fait. Cependant je vous prie de vouloir bien y ajouter encore.

Herodote ${ }^{90}$ et Diod. Sicul. ${ }^{91}$ avec le texte grec

et Appian d'Alexandrie, aussi avec le texte grec, mais si la traduction françoise se ${ }^{92}$ trouve a la bibliotheque je serai charmé de l'avoir aussi ${ }^{93}$.

Je suis avec une parfaite consideration, Monsieur

Votre tres humble et tres obeïssant Serviteur

Le Comte J. Potocki

Brunswick. le. 20. fevrier 1796.

[194v: blanc]

\section{Lettre VIII}

[197r]

Monsieur.

J'ai l'honneur de vous envoyer ci joints tous les livres que j'ai eu de la bibliotheque aussi bien que les cartes. a l'exception de.

89. Note en Kurrentschrift allemande: "mit einem päckel bücher gezeichnet» ["accompagnant un paquet de livres», je traduis].

90. Hérodote, Historiai, éd. non identifiée; trad. Les histoires, éd. non identifiée (Lettre VI). Référence soulignée.

9I. Diodore de Sicile, Bibliothéke historiké, éd. non identifiée; trad. La bibliothèque des histoires, éd. non identifiée (Lettre VI). Référence soulignée.

92. corr. de s'y, biffé.

93. Appien d'Alexandrie, Rhomaika, éd. non identifiée; trad. Histoire romaine, éd. non identifiée. Référence soulignée. 
1. un petit atlas sur velin ${ }^{94}$

2. Une carte sur velin pour etre suspendue ${ }^{95}$

3. Collection des oraisons dominicales ${ }^{96}$.

4. Beauplan Description d'Uckraine ${ }^{97}$.

J'aurai l'honneur de remetre moi même ces ouvrages, en prenant congé de vous.

J. Potocki

Brunswick. le. 1. Mai. 1796.

[197v et $198 \mathrm{r}:$ blancs]

[198v: Adresse et sceau; note postale ${ }^{98}$ ]

[A côté de l'adresse:] item j'ai encore ici les neuf poeteresses [sic] grecques $^{99}$.

\section{Lettre IX}

[195r]

Berlin. le. 14. juin [1796].

Monsieur.

Je viens de recevoir la votre en date du 11. et je vous demande bien des excuses de vous avoir laissé si longtems dans l'incertitude au sujet du livre intitulé Description de l'Uckraine par Beauplan ${ }^{100}$.

94. Seeatlas. Ein Atlas von 14 Doppelblättern plus letztes Blatt aus Pergament, Venise, 1554 (Lettre I).

95. Ottomanno Freducci, Portulankarte des Mittel-Meeres mit ganz Europa und Africa/Nord, s. 1., 1497 (Lettre I).

96. Gustav von Bergmann (Hrsg.), Das Gebeth des Herrn, oder Vaterunsersammlung in 152 Sprachen, Ruien, 1789 (Lettre III).

97. Guillaume le Vasseur de Beauplan, Description d'Ukranie qui sont plusieurs Provinces du Royaume de Pologne, Rouen, 1660 (Lettres V et IX).

98. Note en Kurrentschrift allemande: "nebst einer kässe mit bücher gezeichnet» [«accompagnant une caisse de livres», je traduis].

99. [Alcaeus et al.], Carmina novem illustrium feminarum [...], éd. par Fulvio Orsini, Anvers, 1568 (Lettre I).

IOo. Guillaume le Vasseur de Beauplan, Description d'Ukranie qui sont plusiers Provinces du Royaume de Pologne, Rouen, 1660 (Lettres V et VIII). La référence est soulignée dans la lettre. 
Cet ouvrage est entre les mains de Monseigneur le Duc regnant ${ }^{101}$. Si son Altesse Serenissime ne s'en rappeloit pas, vous pouvés le demander a Monseigneur le Prince hereditaire ${ }^{102}$ qui en est parfaitement informé.

J'espere que la Schul buch handlung ${ }^{103}$ vous aura fait remetre deux exemplaires de mes deux premiers volumes, l'un pour la bibliotheque, et l'autre pour vous même. Le troisieme sera mis en lumiere ces jours ci, et vous pourés le reclamer egalement ${ }^{104}$.

Je suis sur le point de retourner en Pologne ${ }^{105}$, ayés donc la bonté de recevoir ici mes adieux acompagnés des plus sinceres remerciements pour la maniere dont vous m'avés aidé dans mes travaux Slaviques et Sarmates. V. t. H. et.

Le Comte Jean Potocki

[195v et $196 \mathrm{r}$ : blancs]

[196v: Adresse et sceau]

IOI. Karl Wilhelm Ferdinand von Braunschweig-Wolfenbüttel (1735-1806).

I02. Karl Georg August von Braunschweig-Wolfenbüttel (1766-1806).

I03. La «Braunschweiger Buchhandlung» est la maison d'édition fondée par l'écrivain Joachim Heinrich Campe (1746-1818). Il y publiait un grand nombre de ses œuvres pédagogiques. Campe est le gendre de l'imprimeur Friedrich Vieweg à qui Potocki envoie en 1797 un volume des Fragments historiques et géographiques sur la Scythie, la Sarmatie et les Slaves ainsi que le Mémoire sur un nouveau péryple du Pont Euxin (Euvres, V, p. 51). Par l'intermédiaire de Campe, Vieweg transfère en 1799 son imprimerie de Berlin à Brunswick (D. Diestelmann, Kleine Braunschweiger Stadtgeschichte, p. 89).

I04. Fragments historiques et géographiques sur la Scythie, la Sarmatie et les Slaves. Apparemment, Potocki ne recourt pas à l'ordre éditorial ici, mais à l'ordre chronologique de la publication. Le tome III paraît fin 1795, suivi par les tomes I et II durant 1796. Le «troisieme» volume paraîtra bientôt, car le 10 juillet 1796, Potocki transmet à Platon Alex. Zoubov les deux volumes restants en rappelant qu'il lui avait envoyé auparavant «un de [ses] volumes sur l'histoire des Slaves» (Euvres, V, p. 47).

IO5. De Berlin, Potocki part pour Vienne où il se prépare à continuer le voyage fin novembre. Il se retrouve à Varsovie en décembre 1796. 


\section{Lettre X}

[192r]

Monsieur

Permettes que par celle ci, j'aye l'honneur de vous recomander $\mathrm{Mr}$ Twidal Gentilhomme anglois qui veut (ainsi que Mr. Dighby son compagnon dans ce voyage) voir la bibliotheque de Wolfenbutel ${ }^{106}$.

$\mathrm{Mr}$ Twidal a tous les droits possibles a n'etre pas confondu avec le commun des voyageurs et surtout a l'egard des bibliotheques, vu qu'il est un Greciste tres distingué. Je suis charmé d'avoir cette occasion de me récomander a votre souvenir.

Jean Potocki

[sans date]

[192v et $193 \mathrm{r}$ : blancs]

[193v: Adresse et sceau]

I06. On ne trouve pas d'indication concernant d'éventuels emprunts effectués par ces deux Messieurs dans le répertoire établi par M. Raabe (Leser und Lektüre vom 17. zum 19. Jahrhundert). 


\section{ANNEXE II}

\section{LISTE DES OUVRAGES CONSULTÉS PAR JEAN POTOCKI À WOLFENBÜTTEL}

On trouvera entre parenthèses la source de la référence. Le sigle RP renvoie aux registres des prêts (K. Bednarska-Ruszajowa, "Graf Jan Potocki in der Herzoglichen Bibliothek in Wolfenbüttel», p. 30-32), le chiffre romain renvoie aux lettres ci-dessus.

Ouvrages dont l'emprunt, le retour ou la consultation sont attestés

Agnese, Battista, [carte non identifiée], Venise, 1514 (Mémoire sur un nouveau péryple du Pont Euxin, p. 4-5).

[Alcaeus et al.], Carmina novem illustrium feminarum [...], éd. par Fulvio Orsini, Anvers, 1568 (RP, I, VIII).

Apulée, Apuleii Madaurensis Platonici Opera Omnia quae exstant, éd. non identifiée (I, IV).

Aurelius Victor, ouvr. non identifié. (VI).

Assemani, Giuseppe Simone, Kalendaria ecclesiae universae [...], Kalendaria ecclesiae Slavicae sive Graeco-Moscha, Rome, 1755 (I, IV).

Barhebraeus, Specimen Historiae Arabum, Oxford, 1650 (IV).

Beauplan, Guillaume le Vasseur de, Description d'Ukranie qui sont plusieurs Provinces du Royaume de Pologne, Rouen, 1660 (RP, V, VIII, IX).

Bergeron, Pierre de (éd.), Les Voyages Fameux du Sieur Vincent Le Blanc Marceillois [...], Paris, 1649 (IV).

Bergmann, Gustav von (éd.), Das Gebeth des Herrn, oder Vaterunsersammlung in 152 Sprachen, Ruien, 1789 (RP, III, VIII). 
Carpzov, Johann Benedict, Neueröffneter Ehren-Tempel Merckwürdiger Antiquitäten des Marggraffthums Ober-Lausitz, Leipzig/Budissin, 1719 (I, V).

Cassiodore, Variae epistolae, éd. non identifiée (I, V).

Dantine, Maur François, Durand, Ursin, Clémencet, Charles, L'Art de vérifier les dates des faits historiques, des chartes, des chroniques, et autres anciens monumens [...], Paris, 1750 ; nouvelle édition revue et augmentée, Paris, 1770 (RP, I).

Dionysius Halicarnasseus, Dionysii Halicarnassei Scripta omnia quae extant, historica et rhetorica, éd. par Friedrich Sylburg, Francfort, 1586 (RP).

Freducci, Ottomanno, Portulankarte des Mittel-Meeres mit ganz Europa und Africa/Nord, s. 1., 1497 (RP, I, VIII, Mémoire sur un nouveau péryple du Pont Euxin, p. 4).

Leibniz, Gottfried Wilhelm, Scriptores rerum Brunsvicensium, Hannovre, 1707-1711 (I, IV).

Mallet, Paul-Henri, Histoire de Dannemarc, Copenhague, 1755-1765 (IV, VI).

Petrus de Dusburg, Chronicon Prussiae, éd. par Christoph Hartknoch, Francfort/Leipzig/Jena, 1679 (I, III, IV).

Pline l'Ancien, Naturalis historia, éd. non identifiée; trad. Histoire naturelle, éd. non identifiée (IV, VI).

Ptolémée, Claude, Cosmographia, éd. non identifiée; trad. Géographie, éd. non identifiée (IV, VI).

Rivet de la Grange, Dom Antoine, Histoire littéraire de la France [...], éd. par la Congrégation de Saint-Maur, Paris, 1733- (RP).

Schedel, Hartmann, Das buch Der Croniken, Nuremberg, 1493 (RP).

Solinus, Caius, Julius, Polyhistor, éd. non identifiée (IV, VI).

Sylburg, Friedrich (éd.), Historiae Augustae Scriptores Latini Minores [...], Francfort, 1588 (IV, VI).

Symmaque, Quintus Aurelius, Epistolarum libri decem, Leyde, 1653 (V). Valvasor, Johann Weikhard von, Die Ehre deß Hertzogthums Crain [...], Laybach, 1689 (I, III, V).

Zosime, Xiphilin, Zonare, Histoire romaine, trad. par Louis Cousin, Paris, 1686 (VI).

[Auteur non identifié], Seeatlas. Ein Atlas von 14 Doppelblättern plus letztes Blatt aus Pergament, Venise, 1554 (RP, I, VIII, Mémoire sur un nouveau péryple du Pont Euxin, p. 5). 
[Auteur non identifié], Theoremata, éd. non identifiée (V).

Ouvrages dont seule la commande est attestée

Ammianus Marcellinus, Res gestae ou les dixhuit livres de son histoire qui nous sont restés, trad. par Guillaume de Moulines, Berlin, 1775 (VII).

Anastase le Bibliothécaire, Chronographia tripartita, éd. non identifiée (I).

Appien d'Alexandrie, Rhomaika, éd. non identifiée; trad. Histoire romaine, éd. non identifiée (VII).

Avienus, Rufius Festus, Descriptio orbis terrae, éd. non identifiée.

Barhebraeus, Chronicon Syriacum, éd. par Georg Wilhelm Kirsch, Leipzig, 1789 (I).

Bergeron, Pierre de (éd.), Voyages faits principalement en Asie dans les XII, XIII, XIV, et XV siecles, La Haye, 1735 (I).

Byzantinus, Stephanus, Ethnika kat' epitomen, éd. non identifiée (V).

Denina, Carlo, Delle Rivoluzioni d'Italia Libri Ventiquattro, Turin, 1769-1770 ; trad. par Abbé Jardin, Révolutions d'Italie, Paris, 1771-1775 (V).

Diodore de Sicile, Bibliothéke historiké, éd. non identifiée; trad. La bibliothèque des histoires, éd. non identifiée (VI, VII).

Diogène LaËrCe, De vitis, dogmatibus et apophtegmatibus clarorum philosophorum libri $X$, éd. non identifiée; trad. anonyme, Les vies des plus illustres philosophes de l'antiquité [...], Amsterdam, 1758 (I).

Guignes, Joseph de, Histoire générale des Huns, des Turcs, des Mogols et des autres Tartares occidentaux, Paris, 1756-1758 (I).

D’Herbelot de Molainville, Barthélemy, Bibliothèque orientale ou Dictionnaire universel contenant generalement tout ce qui regarde la connoissance des peuples de l'Orient [...], Paris, 1697 (III).

Hérodote, Historíai, éd. non identifiée; trad. Les histoires, éd. non identifiée (VI, VII).

Hippocrate de Cos, De aere, aquis et locis opus, éd. non identifiée (VI). Hudson, John (éd.), Geographiae veteris Scriptores Graeci Minores, Oxford, 1698-1717 (IV).

L'Hermite-Souliers, Jean-Baptiste, Histoire généalogique de la noblesse de Tourraine [...], Paris, 1665 (IV). 
Lenglet-Dufresnoy, Nicolas, Tablettes chronologiques de l'histoire universelle sacrée et profane, ecclésiastique et civile [...], éd. non identifiée (I).

Mariana, Juan de, Histoire générale d'Espagne, trad. par Jean-Baptiste

Nicéphore de Constantinople, Chronographia, éd. non identifiée (I). Paul le Diacre, De gestis Langobardorum libri VI, éd. non identifiée (I).

Paulus Orosius, Adversus paganos Historiarum libri septem, éd. non identifiée (IV).

Pomponius Mela, De situ orbis libri tres, éd. non identifiée (IV).

Ramusio, Giovanni Battista, Navigationi e viaggi [...], éd. non identifiée (I).

Strabon, Geôgraphiká, éd. non identifiée; trad. La Géographie, éd. non identifiée (VI).

Théophane le Confesseur, Chronographia, éd. non identifiée (I).

Thucydide, Historía tou Peloponnesiakoú polémou, éd. non identifiée; trad. Histoire de la guerre du Péloponnèse, éd. non identifiée (VI).

Vossıus, Isaac (éd.), Periplus Scylacis Caryandensis [...] ; Accedit Anonymi Periplus [...], Amsterdam, 1639 (IV).

[Sans auteur], Table Peutigerienne, éd. non identifiée (IV).

[Sans auteur], Itinerarium Antonini Augusti, éd. non identifiée (IV). 


\section{BIBLIOGRAPHIE}

\section{Textes}

Braunschweigische Anzeigen, Brunswick, 27 juillet 1878.

Braunschweiger Tageblatt, Brunswick, 454 (27 septembre 1890).

Gatterer, Johann Christoph, Einleitung in die synchronistische Universaltheorie, Göttingen, 1771.

La Maisonfort, marquis de, Mémoires d'un agent royaliste sous la Révolution, l'Empire et la Restauration 1763-1827, éd. par Hugues de Changy, Paris, Mercure de France, 1998.

Ротоскі, Jean, Chroniques, mémoires et recherches pour servir à l'histoire de tous les peuples slaves. Livre XLII. Comprenant la fin du neuvième siècle de notre ère, Varsovie, 1793.

—, Mémoire sur un nouveau péryple du Pont Euxin, ainsi que sur la plus ancienne histoire des peuples du Taurus, du Caucase, et de la Scythie, Vienne, 1796.

-, Fragments historiques et géographiques sur la Scythie, la Sarmatie et les Slaves, Berlin, 1795 (t. III) et Brunswick, 1796 (t. I, II et IV).

—, Histoire primitive des peuples de la Russie avec une exposition complète de toutes les notions, locales, nationales et traditionnelles, nécessaires à l'intelligence du Quatrième Livre d'Hérodote, Saint-Pétersbourg, 1802.

—, Euvres, 5 t. en 6 vols, éd. par François Rosset, Dominique Triaire, Louvain/Paris, Peeters, 2004-2006 (La république des lettres 11-16).

Küttner, Karl Gottlob, Reise durch Deutschland, Dänemark, Schweden, Norwegen und einen Theil von Italien in den Jahren 1797, 1798, 1799, Leipzig, 1801.

Statuten des grossen Clubbs zu Braunschweig, $3^{\text {e }}$ éd. révisée, Brunswick, Vieweg, 1813. 


\section{Etudes}

Bednarska-Ruszajowa, Krystyna, "Graf Jan Potocki in der Herzoglichen Bibliothek in Wolfenbüttel", in Studien zur Geschichte der deutsch-polnischen Kulturbeziehungen vom Mittelalter bis zum 19. Jahrhundert, hrsg. von Jan Pirożyński, Kraków, Nakładem Uniwersytetu Jagiellońskiego, 1994, p. 23-45 (Zeszyty naukowe Uniwersytetu Jagiellońskiego 1138).

Camerer, Luitgard et al., Braunschweiger Stadtlexikon, Brunswick, Meyer, 1992.

Diestelmann, Dieter, Kleine Braunschweiger Stadtgeschichte, Regensburg, Pustet, 2006.

RAABE, Mechthild, Leser und Lektüre vom 17. zum 19. Jahrhundert: die Ausleihbücher der Herzog August Bibliothek Wolfenbüttel 16641806, München, Saur, 1989-1998; sp: Leser und Lektüre im 18. Jahrhundert: die Ausleihbücher der Herzog August Bibliothek Wolfenbüttel 1714-1799, part. B, t. 1.

RaAbe, Paul (Hrsg.), Handbuch der historischen Buchbestände in Deutschland: Niedersachsen, Hildesheim/Zürich, OlmsWeidmann, 1998.

Schulz, Erich, So sahen wir Braunschweig, Brunswick, Meyer, s.d. [1949].

Ville de Brunswick, site internet de la bibliothèque municipale, www. braunschweig.de/kultur_tourismus/bibliotheken_archive/stadtbibliothek/sammlungen.html [30 avril 2012].

Zimmermann, Paul, Ernst Theodor Langer, Bibliothekar in Wolfenbüttel, ein Freund Goethes und Lessings, Wolfenbüttel, Zwissler, 1883. 
